\title{
Optimal placement of reinforcement in piggyback landfill liners
}

\author{
Marx, D.H. \\ Department of Civil Engineering, University of Pretoria, Pretoria, South Africa* \\ Email: marxdavidh@gmail.com
}

Jacobsz, S.W.

Department of Civil Engineering, University of Pretoria, Pretoria, South Africa

Email: sw.jacobsz@up.ac.za

Phone: +27 124202925

*Corresponding Author

\begin{abstract}
The optimal placement of geogrid reinforcement in clay liners subject to differential settlement was investigated both numerically and with centrifuge modelling. Two unreinforced liners, a liner reinforced at the top-quarter depth, a liner reinforced at the bottom-quarter depth and a double reinforced liner were modelled in the centrifuge. Differential settlement was induced on the model liners by lowering a trapdoor overlain with sand. By considering: 1) the magnitude of differential settlement required to induce micro-cracks in the liners, 2 ) the strain fields across the liners during differential settlement and 3) the distribution of these strain fields, it was found that dividing the available reinforcement equally between the top-quarter and bottom-quarter of the liner, i.e. double reinforcement, represents the optimal reinforcement strategy.
\end{abstract}

Keywords: geosynthetics, geogrid reinforcement, landfill liners, centrifuge modelling, differential settlement

\section{Background}

Several landfills and dump sites in South Africa were constructed before legislation mandating the lining of landfills was published. The current South African legislation requires a composite barrier system consisting of a compacted clay liner (or equivalent) and an HDPE geomembrane for all waste types except construction rubble and spoils (DEA 2013). Consequently, before old landfill sites can be reused, a lining system has to be built on top of the existing waste to prevent further contamination of the environment. This concept is known as a piggyback landfill.

Municipal solid waste is a highly heterogeneous combination of materials with potential for differential and local settlement throughout the waste body (El-Fadel \& Khoury, 2000; Zekkos et al., 2017). Despite its ductility, a clay liner founded on waste, as in the case of a piggy-back landfill, will 
eventually fissure and crack as the underlying waste settles. As these cracks grow, the permeability of the liner will increase until its ability to protect the groundwater from leachate is compromised.

One approach to preserve the integrity of the clay liner during settlement of the underlying waste is the use of geogrid reinforcement. A geogrid can both increase the stiffness of the system, thus decreasing its deflection, and it can inhibits excessive crack growth. Geogrid reinforcing of clay liners has previously been investigated in a geotechnical centrifuge by Viswanadham \& Jessberger (2005), Viswanadham \& Muthukumaran (2007) and Rajesh \& Viswanadham (2009, 2011, 2012). However, limited research has been done to determine the optimal placement of reinforcement in clay liners.

\section{Mechanisms of geogrid reinforcement}

A geogrid can be used to reinforce a clay liner through one of two distinct mechanisms. Firstly, the geogrid can increase the stiffness of the clay liner. In Figure 1a, a section of an unreinforced clay liner and a transformed section of a geogrid-reinforced liner are shown. The increased stiffness of the reinforced liner results in a cross sectional moment of inertia higher than that of the unreinforced clay. Consequently, the reinforced liner will settle, and crack, less than the unreinforced liner under the same load or deflection.

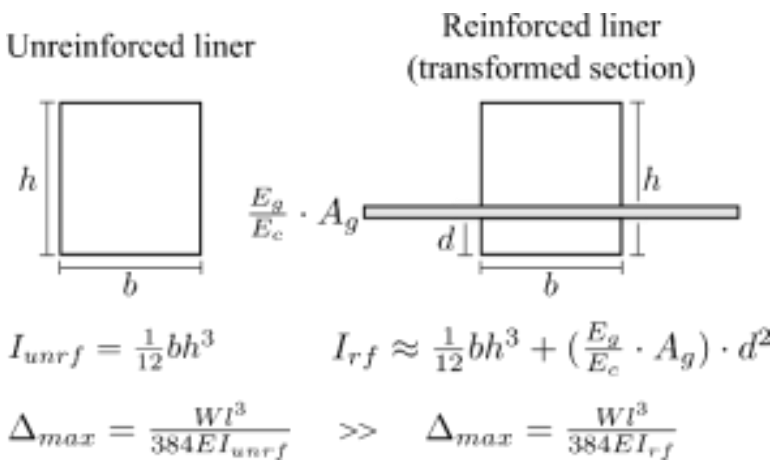

a)

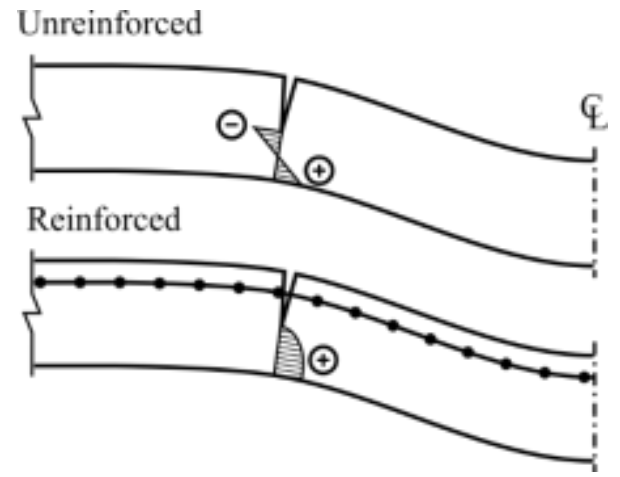

b)

Figure 1 - Mechanisms of geogrid reinforcement: a) increase in stiffness of the system and b) change in stress distribution at crack tip.

The first reinforcement mechanism is independent of the bond between the geogrid and the clay liner. Without any bond, load can still be transferred from the clay to the geogrid below. Consequently, the clay above the geogrid will settle less than when unreinforced. However, the clay below the geogrid might delaminate from the liner and crack.

This use of a geogrid to increase the stiffness of the system is the basis for most geogrid-reinforced liner designs. In these designs the geogrid is placed below the clay liner and is assumed to span over a void as a tensioned membrane (Giroud 1981; Giroud et al. 1990). The geogrid is selected to be both strong enough to prevent collapse of the liner and stiff enough to limit the strain in the clay below its fracture limit.

The second mechanism of geogrid reinforcement depends on the bond between the geogrid and the clay. In Figure $1 \mathrm{~b}$ the stress distribution at the tip of a crack in a bending, unreinforced liner is shown. Due to the crack the neutral axis of the liner moved to centre of the intact section. Consequently, the stress at the tip of the crack remains tensile. As there is a tensile load and a preexisting crack, both components required for crack growth are present (Griffith, 1920). However, when embedding reinforcement in the liner (see Figure $1 \mathrm{~b}$ ) the neutral axis (position of zero strain) moves above the intact section as the tensile stress is now supported by the geogrid. Consequently, 
the intact section of the liner is in compression and the crack will not propagate. Thus, the second reinforcement mechanism serves to change the stress distribution at the crack tip.

Although geogrid reinforcement of clay liners subject to differential settlement have previously been investigated extensively, limited research has been done into the optimal reinforcement position for a geogrid in such a clay liner. The design approach for a reinforced soil layer by Giroud et al. (1990) assumes that the geogrid is placed at the bottom of the liner. However, Viswanadham (1996) recommended placing reinforcement at the top quarter of the liner following the results of centrifuge experiments.

Some recommendations on the placement of reinforcement in granular soils is also available. For a sand layer in a ramp test Palmeira \& Viana (2003) found that the maximum increase in shear strength was obtained by placing a geogrid one third from the base. Similarly Kuo \& Hsu (2003) found the optimal geogrid position in an asphalt overlay to be one third from the base of the road. In repeated load triaxial tests on reinforced granular base material, Abu-Farsakh et al. (2012) found that the lowest permanent deformation occurred when two geogrids were used, one at the top third and one at the bottom third of the sample. For triaxial tests of railway ballast Mishra et al. (2014) found that the maximum increase in shear strength was achieved when two geogrids were used, one placed at $2 / 5^{\text {ths }}$ from the top and one $2 / 5^{\text {ths }}$ from the bottom. Finally, Mousavi et al. (2017) found that the efficiency of a single layer of geogrid reinforcement in unpaved roads measured in terms of surface deformation decreased from $70 \%$ to only $5 \%$ when not placed at the optimal positions in the aggregate base course.

\section{Methodology}

The literature discussion above demonstrates that uncertainty still exists about the optimal placement of geogrid reinforcement in clay liners. As such, the optimal placement of geogrids liner when acting in the first mechanism of reinforcement in a clay liner- increasing the stiffness of the system - was investigated numerically (Marx \& Jacobsz, 2016a, 2016b). A linear elasto-plastic, undrained, Mohr Coulomb finite element model was used. Four levels of geogrid reinforcement (bottom, bottom-quarter, middle and top-quarter depths) were modelled with linear elastic beam elements.

It was assumed that the cost of the reinforcement was represented by the sum of the stiffnesses of the geogrids used at the four positions, e.g. doubling the stiffness was equivalent to doubling the cost. By varying the distribution of the available reinforcement stiffness between the four positions, and calculating the corresponding minimum tensile strain in the liner for a given displacement profile, a Pareto Front was generated (Arora, 2004). The Pareto front defined the minimum magnitude of tensile strain across the full liner as a function of the total reinforcement cost.

An example of a Pareto front from Marx \& Jacobsz (2016b) is shown in Figure 2. In Figure 2a the maximum tensile strain possible in the liner, if the reinforcement is distributed optimally, is shown for a number of total reinforcement costs (sum of geogrid stiffnesses used in the liner), for a given displacement profile. In Figure $2 b$ the optimal distribution of reinforcement for each of these total reinforcement costs is shown. These designs, i.e. the distribution of the available stiffnesses between the four positions, for optimal reinforcement, were defined as the optimal reinforcement strategy (ORS). 


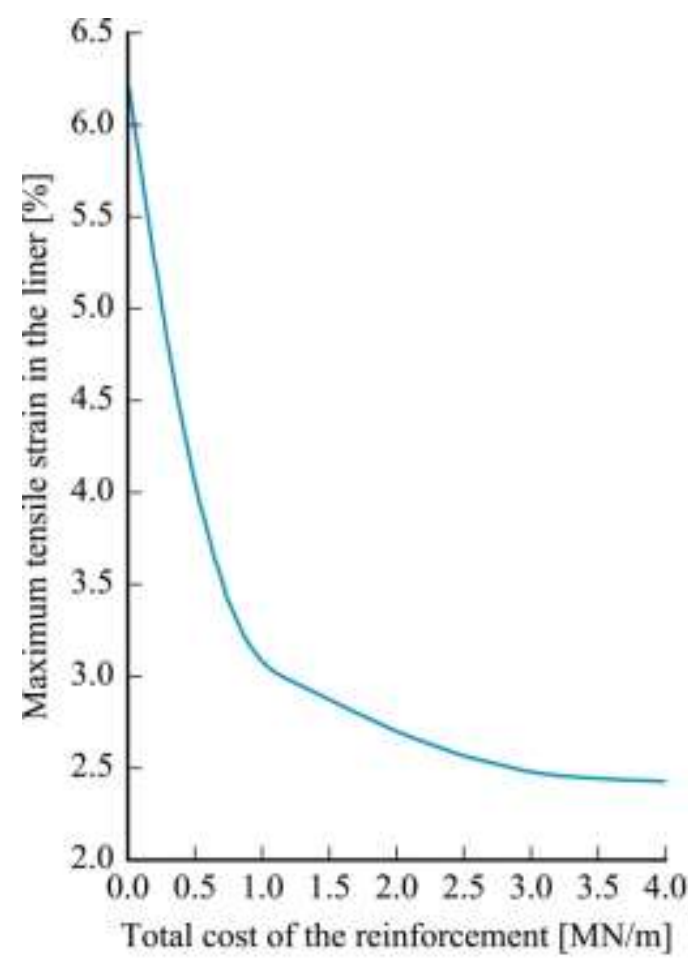

a)

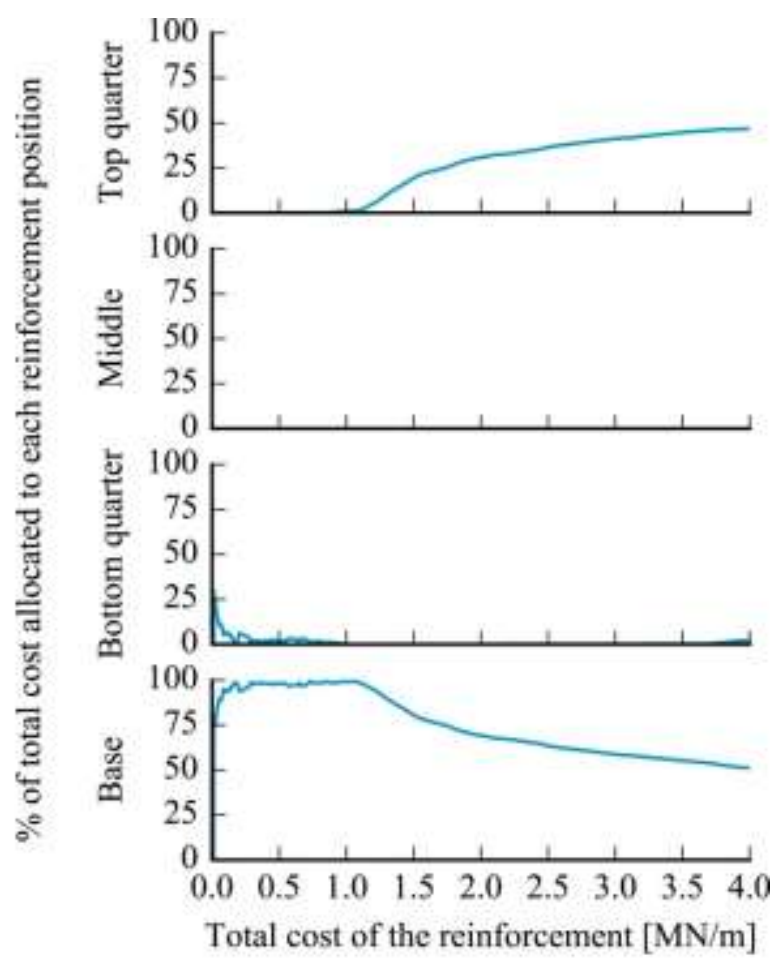

b)

Figure 2 - Pareto Front of tensile strain versus total reinforcement cost a) and Optimal Reinforcement Strategy

It was found, that despite the magnitude of maximum tensile strain varying with a change in liner thickness or overburden pressure, the ORS remained constant for a given displacement profile (Marx \& Jacobsz, 2016b). It can therefore be assumed that when the geogrid acts in the first mechanism, i.e. increasing the stiffness of the system, the behaviour of the reinforced clay liners are independent of liner thickness and overburden pressure. However, the settlement trough geometry and magnitude of central settlement were found to have a significant influence on the optimal reinforcement strategy (ORS). The optimal reinforcement positions were found to be either at the base of the liner, or reinforcement divided equally between the top-quarter and base of the liner, depending on the problem geometry.

By using the results of the numerical analyses as guidance, five centrifuge tests were designed to investigate the optimal placement of reinforcement when the geogrid acts in the second mechanism, i.e. modifying the stress at the crack tip. The two most significant reinforcement positions from the results of the numerical analyses were modelled, i.e. top quarter and bottom quarter depths. As there would not have been sufficient bond between a geogrid at the base of the liner and the clay to suppress crack growth, reinforcement was rather placed at the bottom quarter. No overburden stress was applied to the models as: a) overburden stress did not prove to influence the optimal reinforcement strategy in the numerical analysis (Marx \& Jacobsz, 2016b), b) it allowed for in-test observation of surface crack propagation in plan and, c) it would represent the most critical stage in a liner's life as the addition of overburden stress suppresses tensile crack formation and arguably induces less critical shear ruptures in the liner (Jessberger \& Stone, 1991).

The five centrifuge tests, modelling four different reinforcement strategies, were: 1) two tests of unreinforced model liners used as baselines to compare the reinforced tests against, 2) one model liner reinforced at the top quarter position, the reinforcement strategy deemed optimal by Viswanadham (1996) and Rajesh \& Viswanadham (2009), 3) one model liner reinforced at the bottom quarter position, representing the optimum for the numerical analysis of a liner subject to 
an imposed Gaussian shaped settlement trough and 4) one model liner reinforced both at the top quarter and the bottom quarter positions, i.e. a double reinforced liner. This final strategy was found to be the ORS from the numerical analysis for a liner subject to a generalised bell curve shaped trough shape (Marx \& Jacobsz, 2016b).

\section{Experimental setup}

The 150 g-ton centrifuge of the University of Pretoria (Jacobsz et al., 2014) was used for centrifuge modelling of a reinforced clay liner subject to differential settlement. A $600 \mathrm{~mm}$ long centrifuge strongbox was used. At the test acceleration of $30 \mathrm{~g}$ an $18 \mathrm{~m}$ long prototype liner was thus modelled. This allowed for a fairly large segment of a landfill to be represented by the model.

The centrifuge models consisted of three key components: 1) a reinforced clay layer, 2) on top of a sacrificial sand layer and 3) overlaying a hinged trapdoor used to impose differential settlement of the sand. A front view of the model is presented in Figure 3.

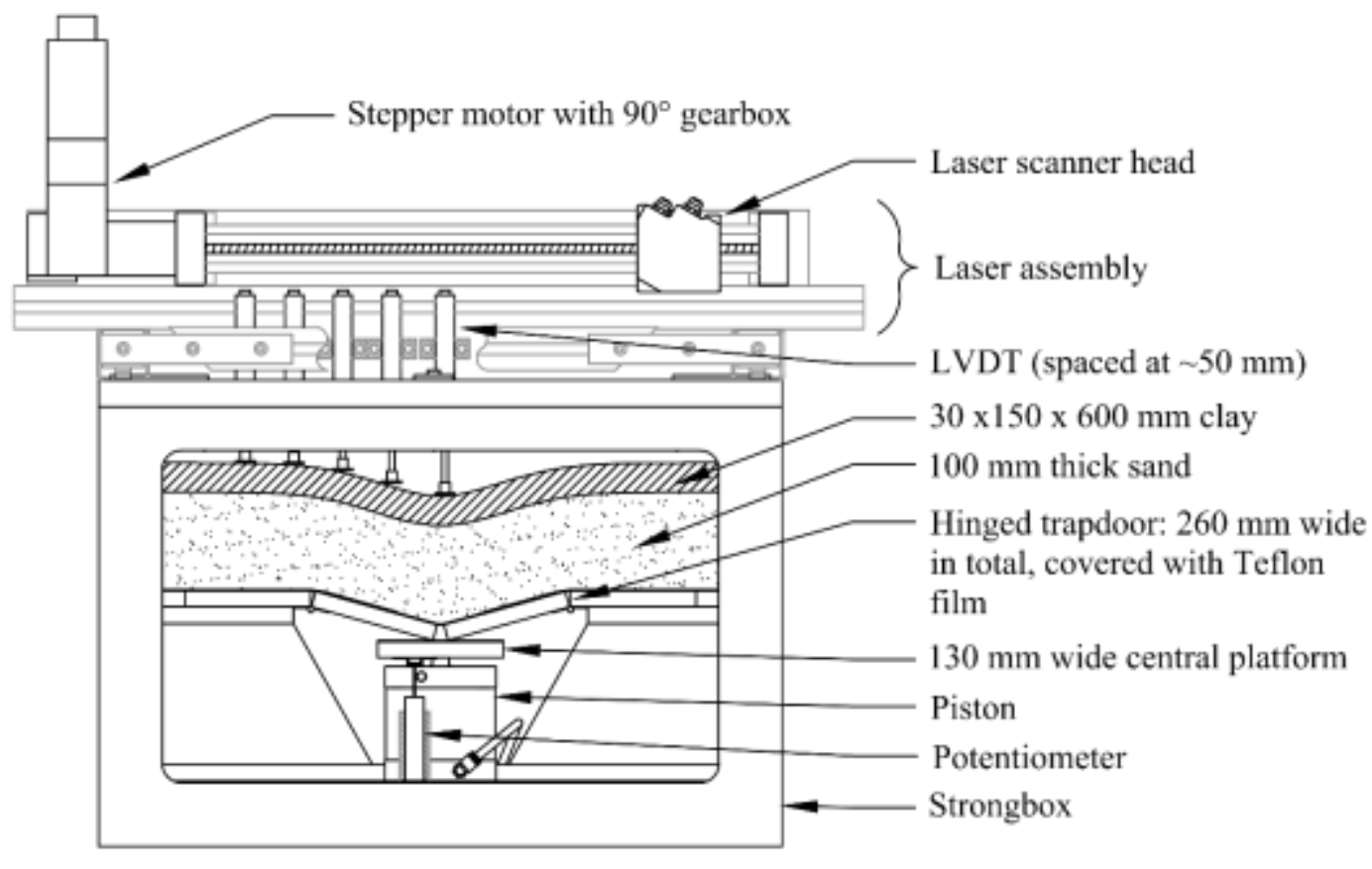

Figure 3 - The centrifuge model.

Model clay liners were trimmed to a thickness of $30 \mathrm{~mm}$ based on practical considerations modelling a $900 \mathrm{~mm}$ thick prototype liner. This thickness falls between the South African standard $(600 \mathrm{~mm}$, DEA (2013)) and liners used in other studies (1200 mm by Viswanadham \& Muthukumaran (2007)).

The model clay liners were $600 \mathrm{~mm}$ long and $150 \mathrm{~mm}$ wide. A glass window in the front and the divider wall within the strongbox was used to confine the model liner to plane strain behaviour. The properties of the reinforcement used in the clay, as well as those of the clay, are discussed in the next section. Below the clay a $100 \mathrm{~mm}$ thick sacrificial sand layer was placed. The purpose of this layer was to smooth the deflected profile imposed by the hinged trapdoor arrangement following Viswanadham \& Muthukumaran (2007).

The shape and width of the imposed settlement trough proved to influence the ORS (Marx \& Jacobsz, 2016b). However, testing models subjected to different shaped settlement troughs fell outside the scope of this study as the focus was on varying the reinforcement positions. Accordingly, the imposed settlement trough shape and width were kept constant. 
A relatively wide settlement trough was modelled, simulating either a localised void forming deep below the liner, or general differential settlement due to waste decomposition. This was done as a local void was judged unlikely to occur near the surface of the old waste dump in a piggy-back landfill. Any porous or weak spaces in the waste surface are likely to be discovered and compacted during preparation of the surface for construction of the new piggy-back liner.

The trapdoor consisted of two $130 \mathrm{~mm}$ wide aluminium plates, supported in the middle of the model on a $130 \mathrm{~mm}$ wide central platform that could be lowered by means of a piston (see Figure 3). At 30g this arrangement allowed a $7.98 \mathrm{~m}$ wide, approximately Gaussian shaped, settlement trough to be imposed. A Teflon sheet covered the aluminium trapdoors to prevent the sacrificial sand from clogging the hinges. Another, covering the central platform, was used to reduce friction between the edges of the trapdoor segments and the central piston.

The central platform was lowered by extracting water from the bottom compartment of the piston. For the first $5 \mathrm{~mm}$ of central settlement the piston was lowered at a rate of $1 \mathrm{~mm} / \mathrm{min}$. This allowed for a future study of arching in the sand at the small movements. For the remainder of the test, the platform was lowered continuously at a rate of $3 \mathrm{~mm} / \mathrm{min}$ to ensure that the behaviour remained undrained. A maximum of $50 \mathrm{~mm}$ of central settlement, or $1.5 \mathrm{~m}$ at prototype scale, could be modelled.

A Canon 100D SLR camera, fitted with a $40 \mathrm{~mm}$ fixed lens, took regular photos of the liner at 6 second intervals as it deformed. These photos were subsequently used to measure the strain in the liner with Digital Image Correlation (DIC) (Stanier et al., 2015).

\section{Materials used}

The model geogrid used to reinforce the model clay liners had to replicate the behaviour of a prototype (full scale) geogrid when under increased acceleration. Arguably, the four fundamental properties governing the behaviour of a geogrid are: stiffness, bond, strength and durability. The durability of the model geogrid is of no concern during centrifuge modelling as tests rarely last longer than a few hours.

The expected tensile strain of a clay liner at failure of 1-3\% (Ajaz \& Parry (1975), Edelmann et al. (1996), Thusyanthan et al. (2007), Gourc et al. (2010), Plé et al. (2012)) is significantly lower than the ultimate tensile strain of a geogrid ( $10 \%$ and greater, e.g. Shinoda \& Bathurst (2004)). Thus, the strain behaviour of the model geogrid only had to be equivalent to the prototype up to the failure strain of the clay as by that stage, the liner would have cracked and failed. Consequently, neither failure strain nor strength were considered when a suitable model geogrid was selected.

Zornberg et al. (2017) showed using a parameter defined as the stiffness of the soil-geosynthetic composite that both confined stiffness and interface shear strength (a function of bond) are required to describe the behaviour of reinforced granular materials at small strain. Consequently, the remaining two fundamental geogrid properties, stiffness and bond, were of importance when selecting a model geogrid for centrifuge testing.

A polyethylene terephthalate (PET) Huesker $\mathrm{HaTe}^{\circledR} 23.142$ mesh was selected for the model geogrid. Properties of the mesh are shown in Table 1. The stiffness of the mesh was tested using the ASTM standard D6637-11 (2011) as guideline. The HaTe ${ }^{\circledR}$ mesh had some slack after being clamped in the grips of the press. Consequently, the sample was firstly strained to a pre-tension load of $2.5 \mathrm{~N}$. After the pre-tension load was reached, the sample was tested at a rate of $6 \mathrm{~mm} / \mathrm{min}$. This strain rate of $12.5 \% / \mathrm{min}$ is within the limit of $10 \pm 3 \% / \mathrm{min}$ required by the standard and the pre-tension load was within the required $1.25 \%$ of the total load. 


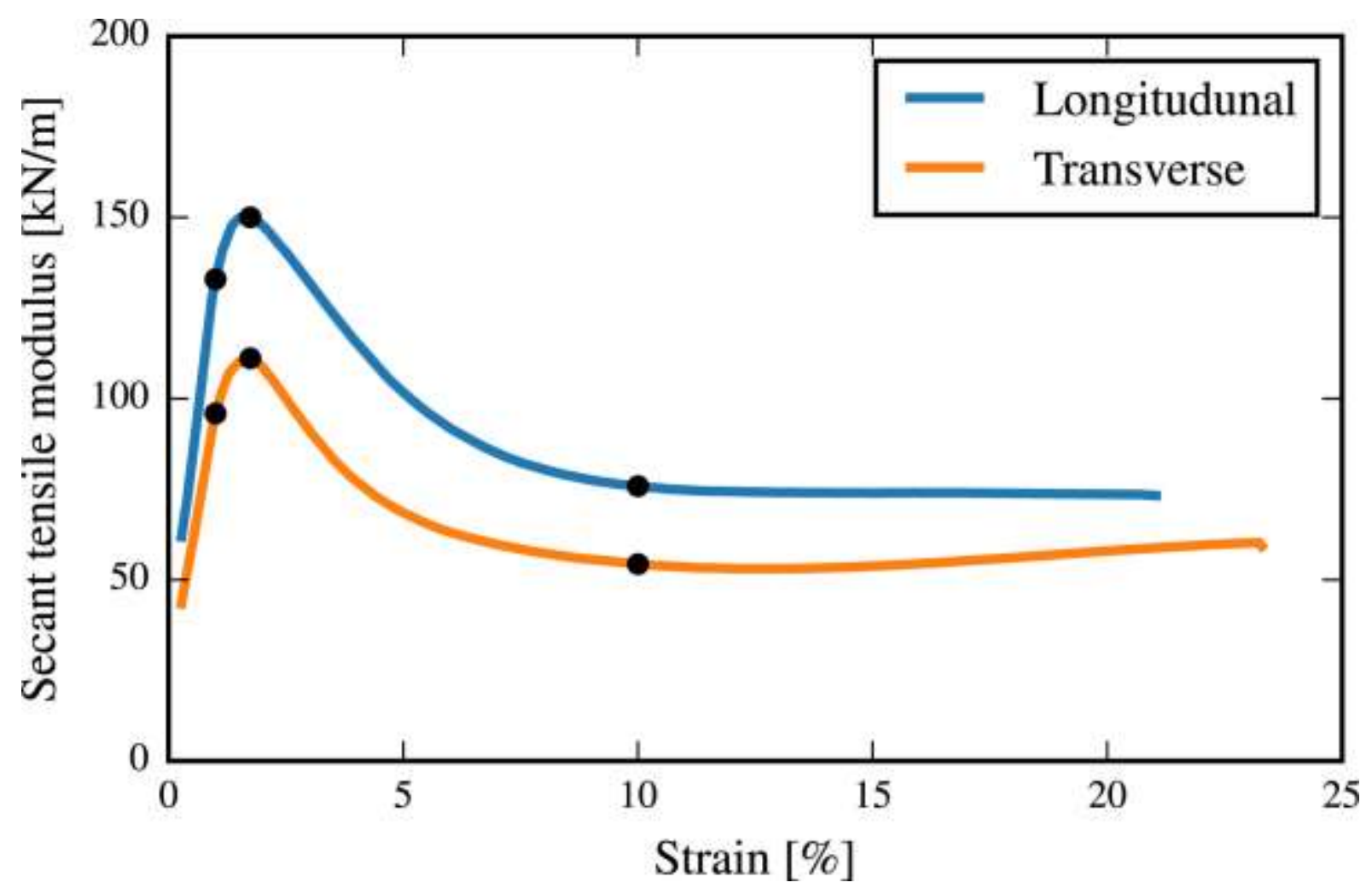

Figure 4 - Secant tensile modulus of the $\mathrm{HaTe}^{\circledR}$ mesh as a function of strain.

Table 1 - Properties of the $\mathrm{HaTe}^{\circledR}$ mesh used as model geogrid.

\begin{tabular}{lrr}
\hline Property & \multicolumn{2}{c}{ Value } \\
\cline { 2 - 3 } & $1 \mathrm{~g}$ & $30 \mathrm{~g}$ \\
\hline Aperture size [mm] & $3 \pm 0.5$ & $90 \pm 15$ \\
Open area [\%] & 64 & 64 \\
Rib thickness [mm] & & \\
Longitudinal & 0.29 & 8.7 \\
Transverse & 0.35 & 10.5 \\
Rib width [mm] & & \\
Longitudinal & 0.61 & 18.3 \\
Transverse & 0.91 & 27.3 \\
Secant stiffness (transverse) [kN/m] & & \\
1\% strain & 95.88 & 2876 \\
1.74\% strain & 111.1 & 3333 \\
10\% strain & 54.31 & 1629 \\
\hline
\end{tabular}

The measured secant tensile modulus-strain curve for the mesh is shown in Figure 4. Initially the tensile modulus increased to a peak at $1.7 \%$ strain. Thereafter, the tensile modulus decreased and eventually plateaued. Selected tensile modulus values are also provided in Table 1.

The equivalent tensile modulus of the model geogrid at $30 \mathrm{~g}(2876 \mathrm{kN} / \mathrm{m}$ at $1 \%$ strain) is high compared to geogrids typically used in practice. However, the use of this mesh as a model geogrid is justifiable for the following three reasons: 1) geogrids with a higher tensile modules have been used in practice to span voids (e.g. $26000 \mathrm{kN} / \mathrm{m}$ at $1.8 \%$ strain by Alexiew (1997)), 2) the failure 
mechanism that occurs will still be representative of a PET geogrid and 3) successful previous centrifuge studies used model geogrids of similarly high tensile modulus (Viswanadham \& Jessberger, 2005). The geogrid was used in the transverse direction and was anchored to the edges of the clay layer to prevent pull-out failure from occurring.

The final property of relevance when selecting the model geogrid for the centrifuge model was bond. The bond of a geogrid is a collective term encompassing the properties that affects pull-out resistance: a) skin friction and b) bearing resistance of the transverse members; and those that affects shear resistance: c) soil-to-reinforcement shear resistance and d) soil-to-soil shear resistance at the grid openings (Alfaro et al,. 1995). As such, the bond of the geogrid is a function of the rib geometry, percentage open area, rib skin friction and geogrid and clay stiffness.

The skin friction of the model geogrid will be representative of prototype geogrids if it is made of one of the typical polymers used for geogrids. The percentage open area of the model geogrid (see Table 1) is relatively low, as with most high-strength prototype geogrids, and might result in premature delamination of the clay liner. Similarly, the thickness of the model geogrid ribs is fairly substantial compared to typical geogrids used in practice. However, Palmeira \& Milligan (1989) found that the size of the transverse members does not influence the pull-out capacity of the geogrid when the ratio between member size and average particle diameter was greater than 12 . Similarly, Stathas et al. (2017) found that there was little difference between the interface friction angle of a $0.2 \mathrm{~mm}$ thick and a $0.5 \mathrm{~mm}$ thick model geogrid tested in a poorly graded sand with ${ }_{50}=0.6 \mathrm{~mm}$. Consequently, the large rib thickness was not regarded to be a concern as the model geogrid was used in a clay.

Finally, Zornberg et al. (1997) and Viswanadham \& König (2004) postulates that for equivalent bond the model geogrid should be $N$ times stiffer than the prototype if the interface behaviour is dictated by the shear-stress - shear-displacement behaviour of the geogrid, rather than shear-stress shear-strain behaviour. As such, the high stiffness of the model geogrid used might have resulted in bond more representative of a prototype geogrid.

Skin friction, transverse member properties, aperture size and stiffness of a model geogrid can all be individually selected to ensure equivalent behaviour to a full-scale geogrid at increased acceleration. Only recently had Stathas et al. (2017) managed to 3D print model geogrids that accurately scaled the tensile strength-strain behaviour of prototype geogrids and achieved representative interface friction angles in granular material. For this study a model geogrid was selected that sufficiently mimicked geogrids in practice to accurately study the mechanism of geogrid reinforcement, if not replicating it exactly.

The clay used in this study was a water washed kaolin powder from the Gujurat province of India. The specific gravity of the kaolin was 2.662 as measured by an AccuPyc II 1340 Pycnometer. A Malvern Mastersizer 2000 particle size analyser was used to measure the particle size distribution (PSD) of the clay shown in Figure 5. The Atterberg limits of the kaolin were as follows: a liquid limit (LL) of $37 \%$ and a plasticity index (PI) of $9 \%$. This liquid limit is fairly low compared to the liquid limits for the kaolin used for model liners in literature (42\% to 61\%: Schofield \& Wroth 1968; Koutsourais et al. 1991; Viswanadham, 1996; Viswanadham \& Mahesh, 2002; Thusyanthan et al., 2005; Lehane et al., 2009). However, the low liquid limit appears to be common for kaolin clays in general (Casagrande, 1932) and might be related to the relatively coarse particle size or incomplete hydration of micro-clods. 


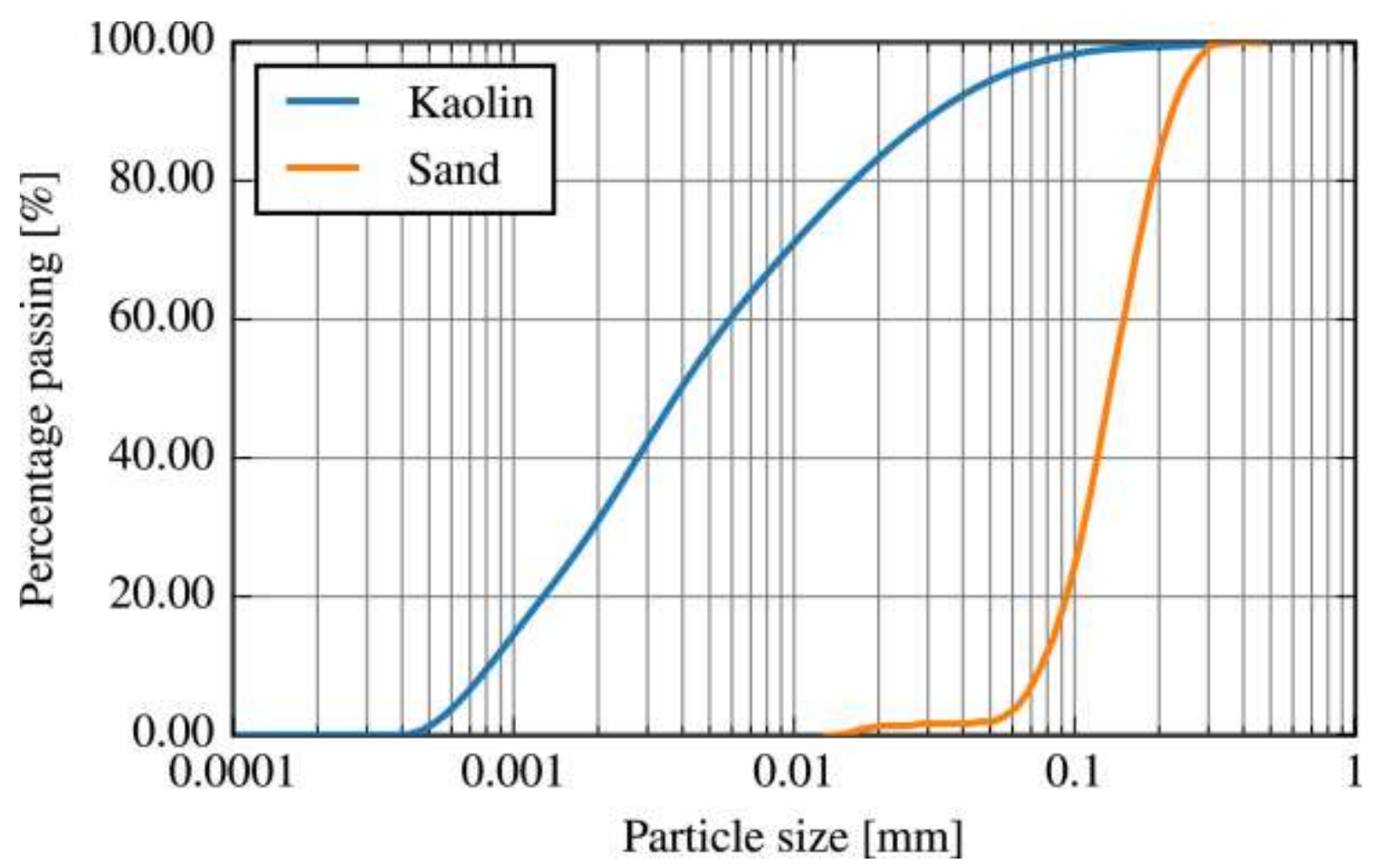

Figure 5 - Particle size distribution of the kaolin clay and sacrificial sand.

The clay was consolidated in stages to $610 \mathrm{kPa}$ resulting in a dry density of $1416 \mathrm{~kg} / \mathrm{m}^{3}$. This density is equivalent to the $95 \%$ of the Proctor density for the clay, corresponding to a void ratio of 0.88 . The reinforced liners were consolidated in multiple lifts. Firstly, the bottom clay was consolidated. Thereafter the geogrid was placed, additional clay slurry was poured and the consolidation was repeated. After the model liners were cut to the required size, sand was embedded on the side facing the camera to provide texture for the DIC analysis.

A comprehensive investigation into the properties of the sacrificial sand layer used was done by Archer \& Heymann (2015). The grading curve of the sand is shown in Figure 5 . The specific gravity was 2.666 , the minimum void ratio 0.60 and the maximum void ratio 0.92 .

\section{Results}

The effect of reinforcement position on the behaviour of the model liners was evaluated in terms of the central settlement required for micro-crack growth, the shear strain concentrations that formed in the liners, and the distribution of horizontal and shear strain through the liners.

\section{Micro crack formation}

When a liner bends due to differential settlement, tensile stress is generated in the clay. As the tensile stress increases, micro-cracks grow from existing defects in the fracture process zone, progressively weakening the clay. Eventually the material will be weak enough for the micro-cracks to aggregate into a macro-crack and the two surfaces of the crack will separate (Karihaloo, 1995).

The emergence of micro- and macro-crack growth in a liner can be identified from the tensile loaddeflection curve of a material. In Figure 6 two possible tensile load-deflection curves are shown. From $\mathrm{O}$ to $\mathrm{A}$ the behaviour is linear. Once micro-cracking damages the material $(A)$, it progressively supports a smaller load increment for every displacement increment (i.e. the behaviour becomes non-linear). Finally, the tensile stress reaches a peak (B) and macro-cracks opens (Turner \& Kolednik, 
1994; Karihaloo 1995). Post-peak the load can either plateau for a plastic material (B-C) or drop for a quasi-brittle material (B-D).

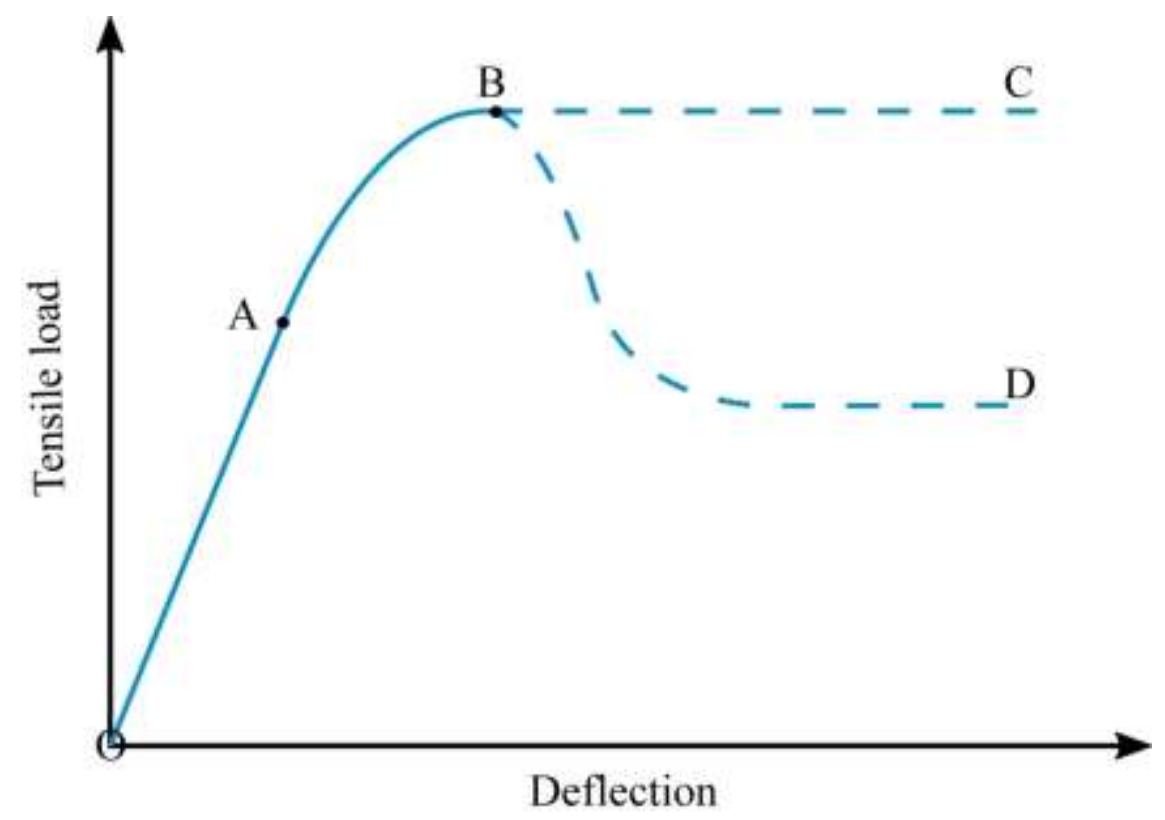

Figure 6 - Typical tensile load-deflection curve of a material that cracks. B-C is an elastic-plastic material while $B-D$ is a quasi-brittle material.

The locations where macro cracks formed along the length of the model liners were identified from the photos taken. At each of these locations the linear horizontal strain at the side of the liner facing the glass window was calculated from the displacement field of the clay as measured by the DIC analysis. In principle, a DIC analysis entails measuring the displacement of patches (in this case measuring $16 \times 16$ pixels) of soil between successive digital images. For an in depth discussion of the technique see White et al. (2003) and Stanier et al. (2015). The GeoPIV-RG software of Stanier et al. (2015) was used for these analyses.

The evolution of linear horizontal strain along the top edge of the liners where macro-cracks were observed in the liners are shown in Figure 7. Cracks opened across three different locations in each liner: the left hogging zone, the centre sagging zone and the right hogging zone of the liner. The settlement of the central piston represents the magnitude of the differential settlement of the sand. Markers indicates where the strain-settlement behaviour changed from linear to non-linear which is hypothesized to coincide with micro-cracks opening up (Turner \& Kolednik, 1994; Karihaloo 1995). These relevant central settlement magnitudes are summarised in Table 2.

The unreinforced liner cracked at a smaller central settlement $(4.06 \mathrm{~mm})$ than the bottom-quarter reinforced liner that, in turn, cracked at a smaller central settlement $(7.88 \mathrm{~mm})$ than the top-quarter $(12.7 \mathrm{~mm})$ and double reinforced $(22.7 \mathrm{~mm})$ liners. Thus, under the settlement trough imposed, the top-quarter and double-reinforced liners could withstand greater differential settlement of the sand before the integrity of the liners was compromised, compared to the bottom-quarter reinforced liner. All three reinforced liners could withstand greater differential settlement than the unreinforced liner, illustrating the benefit of geogrid reinforcement. However, two model geogrids were used to reinforce the double-reinforced liner. Therefore, the total stiffness of the reinforcement used in this liner was double that of the others. To directly compare the results from a single reinforced test with a test with double the amount of reinforcement, a suitable point of comparison had to be found. 


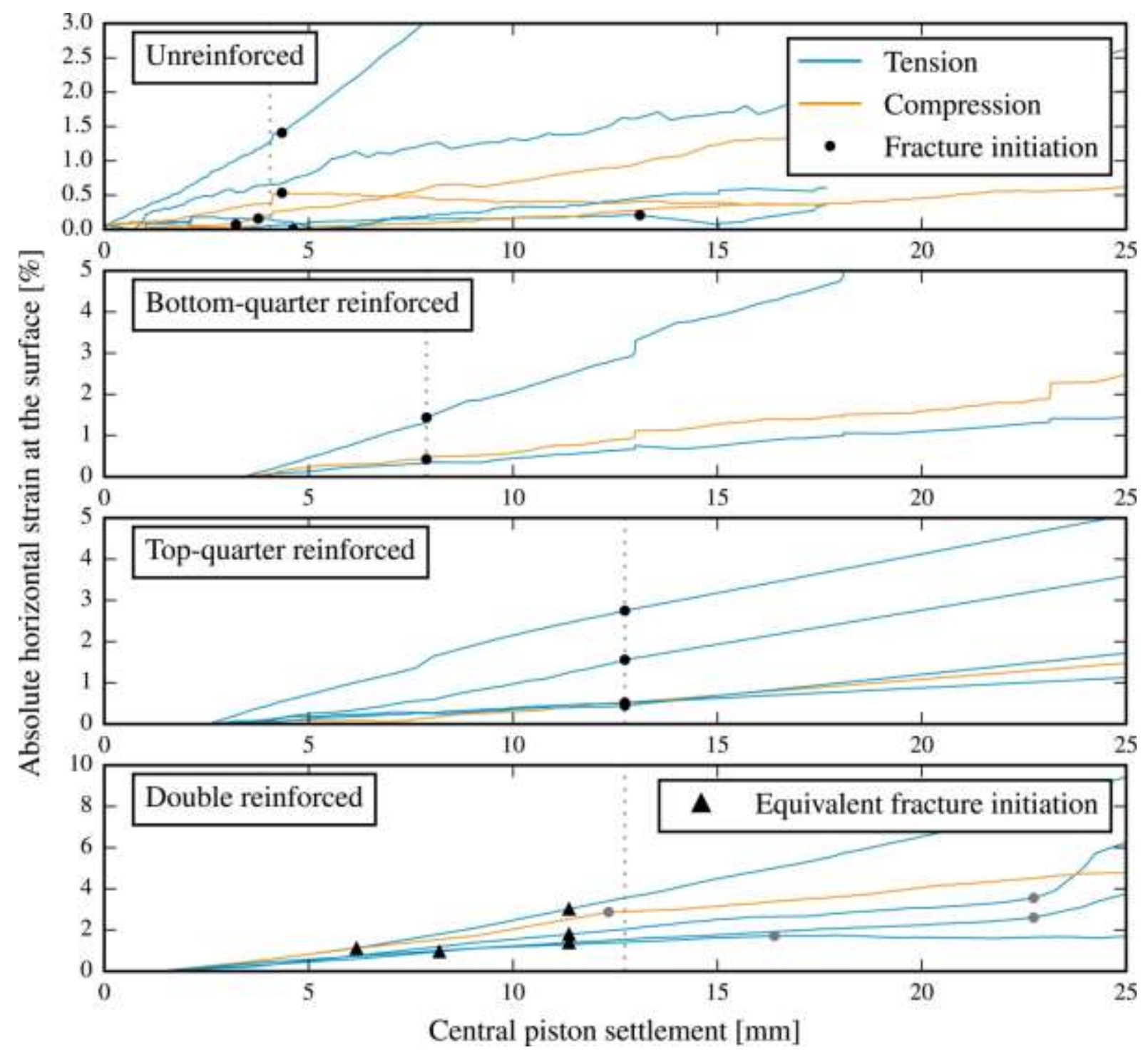

Figure 7- Development of horizontal surface strain with central piston settlement at the locations where cracks were observed. The hypothesized initiation of micro-cracks is indicated by a marker.

Table 2 - Critical settlement stages in the centrifuge tests for the comparison of the results.

\begin{tabular}{|c|c|c|}
\hline Central settlement stage & Magnitude [mm] & Significance \\
\hline 1 & 4.06 & $\begin{array}{l}\text { Occurrence of micro-cracks in the } \\
\text { unreinforced liner }\end{array}$ \\
\hline II & 7.88 & $\begin{array}{l}\text { Occurrence of micro-cracks in the } \\
\text { bottom-reinforced liner. }\end{array}$ \\
\hline III & $12.7^{\mathrm{i}}$ & $\begin{array}{l}\text { Occurrence of micro-cracks in the } \\
\text { top-reinforced (and double- } \\
\text { reinforced) liner. }\end{array}$ \\
\hline
\end{tabular}

'The equivalent behaviour for the double reinforced liner was at double this central piston settlement.

In order to determine the point of comparison, the behaviour of the reinforced clay liners was assumed to be linear elastic until the onset of micro-cracks. To compare the behaviour of a system reinforced with a single a geogrid at quarter depth (Liner A) to that of a liner reinforced at both quarter depths (Liner B), i.e. double the reinforcement stiffness, the point of comparison was selected such that the stress in the stiffer liner $\left(\sigma_{B}\right)$ equalled that in the less stiff liner $\left(\sigma_{A}\right)$ : 


$$
\sigma_{A}=\sigma_{B}
$$

As the most significant stress in the liners is due to bending, the equation above can be expressed as:

$$
\frac{M_{A} y}{I_{A}}=\frac{M_{B} y}{I_{B}}
$$

where $M_{A}$ and $M_{B}$ are the bending moment in the respective liners, $y$ the distance from the neutral axis and $I_{A}$ and $I_{B}$ the moment of inertia of the liners.

When the sand settles differentially a void can potentially form below the liner, and thus support is removed below the liner. Consequently, the self-weight of the unsupported liner segment across the void induces a bending moment in the liner. It is shown in Appendix A that under elastic conditions there is a linear relationship between the central settlement $(\Delta)$, representative of the void size, and moment in the centre of the liner $(M)$, (i.e. $M=k \cdot \Delta)$. Thus:

$$
\begin{gathered}
\frac{k \Delta_{A} y}{I_{A}}=\frac{k \Delta_{B} y}{I_{B}} \\
\Rightarrow \frac{\Delta_{A}}{\Delta_{B}}=\frac{I_{A}}{I_{B}}
\end{gathered}
$$

For the double reinforced (Liner $A$ ) and top- and bottom-quarter reinforced liners investigated in this study, $\Delta_{A} / \Delta_{B}$ simplifies to between 1.2 and 1.7 depending on the strain level. The kaolin had a constrained modulus of $3 \mathrm{MPa}$ at $1.7 \%$ strain measured in the oedometer during swell and the stiffness of the model geogrids used is shown in Table 1. For typical geogrids used in practice $\Delta_{A} / \Delta_{B}$ could be as low as 1.03. In these cases the geogrid will only improve the behaviour of the liner by suppressing crack growth (mechanism 2) and has little influence on the stiffness of the system (mechanism 1).

For the model liners investigated in the present study the maximum tensile stress in the double reinforced liner (Liner B) will equal that in the single reinforced liner (Liner A), when the former settled by 1.2 to 1.7 times more than the lower stiffness system. This is based on the assumption that the behaviour of the liner remains linear-elastic. However, as $\Delta_{A} / \Delta_{B}$ is strain dependent a conservative value of 2 was selected. Thus, for a given settlement of the top- or bottom-quarter reinforced liners, the comparable behaviour of the double-reinforced liner was extracted at double their settlement, as represented by the displacement of the central piston.

The triangle markers in Figure 7 indicate the equivalent settlements for the onset of micro-crack formation in the double reinforced liner had its total reinforcement stiffness been similar to that of the top-quarter and bottom-quarter reinforced liners. At three of the five crack initiation locations the adjusted settlement required for micro-crack formation in the double reinforced liner was similar to that for the top-quarter reinforced liner.

The three settlements stages at which micro-cracks initiated in the unreinforced, bottom-quarter reinforced and top-quarter reinforced liners, i.e. (I) $4.06 \mathrm{~mm}$, (II) $7.88 \mathrm{~mm}$ and (III) $12.7 \mathrm{~mm}$, were selected as points of comparison for subsequent analysis of the centrifuge tests. A summary of these settlement stages is presented in Table 2. In the remainder of the article the settlement stages are respectively referred to as Stage I, Stage II and Stage III. 


\section{Comparison of shear strain concentrations in the reinforced liners}

The GeoPIV-RG DIC software (Stanier et al., 2015) was used to calculate strain fields across the depth of the model liners as they deformed. During the initial acceleration of the centrifuge there was slight settlement of the liners due to slack in the hydraulic system. Consequently, results of the DIC analyses presented are only from where the systems reached equilibrium at $30 \mathrm{~g}$ and excluded the initial distortion of the liners.

The horizontal strain across the liners was calculated and subsequently scaled to fall in the range $[0 ; 1]$. The strains were scaled by the minimum and maximum strain values from Stage III as these were assumed to represent the extremes for the tests. Strain calculated from DIC displacement is prone to some error due to so-called "wild" displacement vectors where incorrect displacements are measured. Consequently, to identify the strain minima and maxima for scaling, the outliers first had to be removed from the data. The statistical distributions of the shear strain values were not normally distributed and thus maximum strain $\left(\varepsilon_{\max }\right)$ values were identified using the skewed boxand-whisker plot as proposed by Hubert \& Vandervieren (2008), taken from the field of Robust Statistics (Rousseeuw \& Hubert, 2011).

Horizontal strain fields across the left hand hogging zone of the model liners are shown in Figure 8. The unreinforced liners simply cracked at both hogging zones, from the top through to the bottom, as reflected in the severe strain concentrations. Similar behaviour was observed in the bottomquarter reinforced liner, with the crack extending from the liner surface to the level of the geogrid. As for the strain-settlement graphs in Figure 7, the double-reinforced and the top-quarter reinforced liners behaved in a similar manner. In both liners the crack terminated at the level of the top geogrid. After the centre displacement was subsequently increased, the cracks propagated through to the bottom. Similar cracking patterns were reported by Jessberger \& Stone (1991) for unreinforced tests and Viswanadham \& König (2004) and Rajesh \& Viswanadham (2009) for reinforced tests. The strain concentration at the base of the left hand section of the double reinforced liner is an artefact of the DIC analysis due to reflections from the glass window and poor texturing - at the end of the test no cracks were visible at this location.

A geogrid provides tensile resistance across the mouth of a cracks, reducing the tensile stress and preventing the crack from opening up. Consequently, the strain concentrations stopped the level of the top geogrid in the top-quarter and double reinforced liners. In contrast, catastrophic crack growth occurred in the bottom-quarter reinforced liner before the additional tensile resistance could be activated. Thus, in effect this liner behaved similar to an unreinforced liner. However, it was not expected that strain concentration in the bottom-quarter reinforced liner would be more severe than for the unreinforced liner. This behaviour is most probably the result of imperfect sample preparation that resulted in preferential paths for the macro-cracks. For central settlement greater than Stage III settlement, the strain concentrations and cracks at the right hand side of the bottomquarter reinforced liner (not shown) were, as expected, less severe than for the unreinforced liner. 

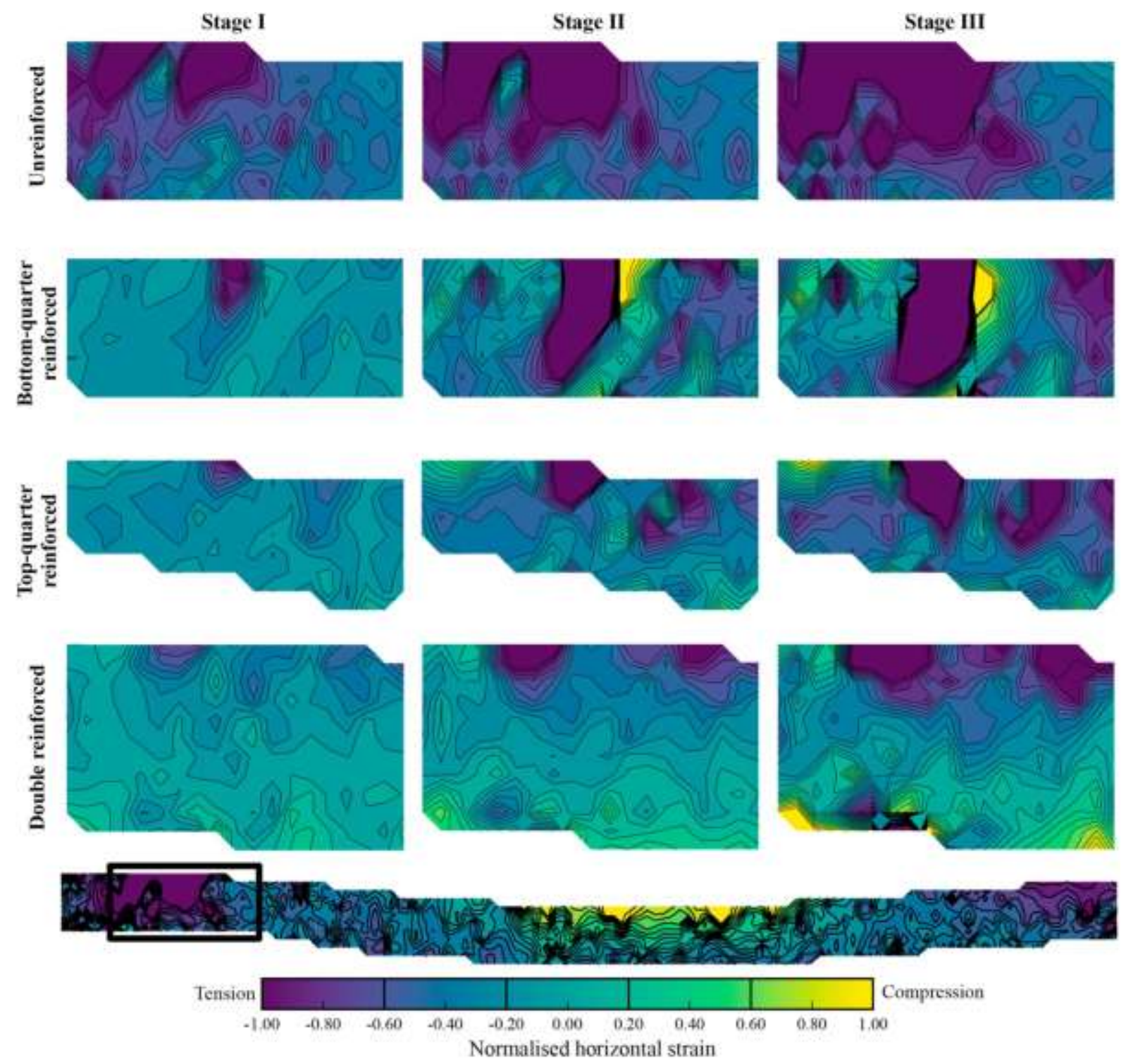

Figure 8 - Comparison of normalised maximum shear strain at the location of the left hand crack for all the tests, at the three different stages (the full unreinforced beam during Stage III is shown at the bottom).

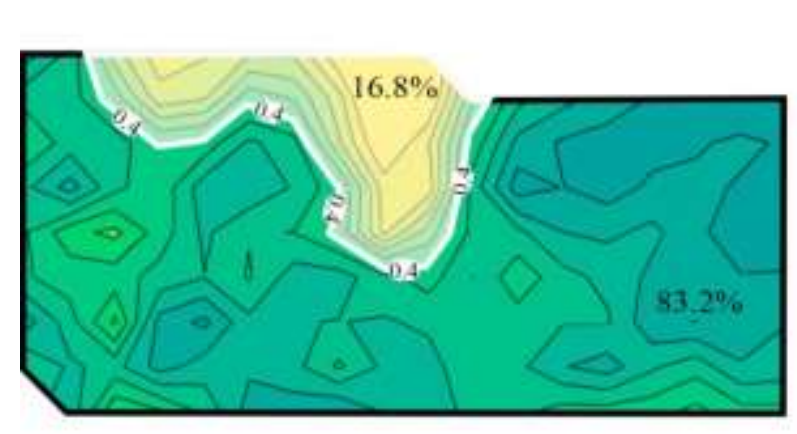

a)

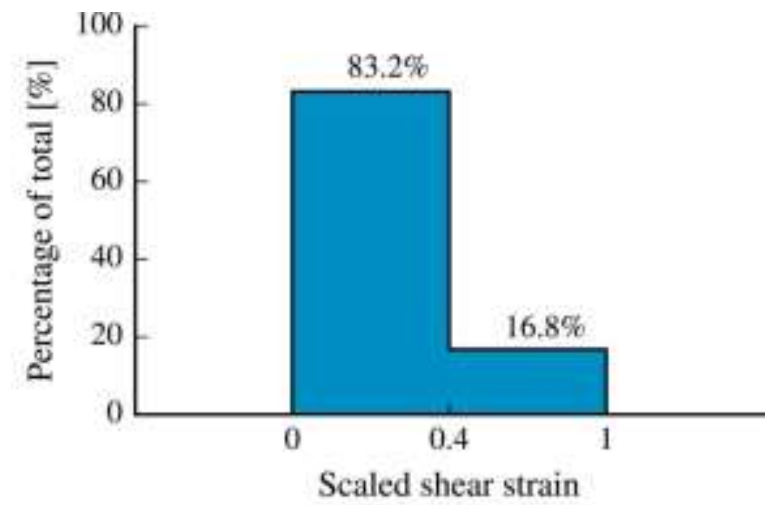

b)

Figure 9 - Generation of the scaled maximum shear strain histograms: a) scaled shear strain field with the area at a strain less than 0.4 indicated and b) equivalent strain distribution indicated on a histogram 
Stage I

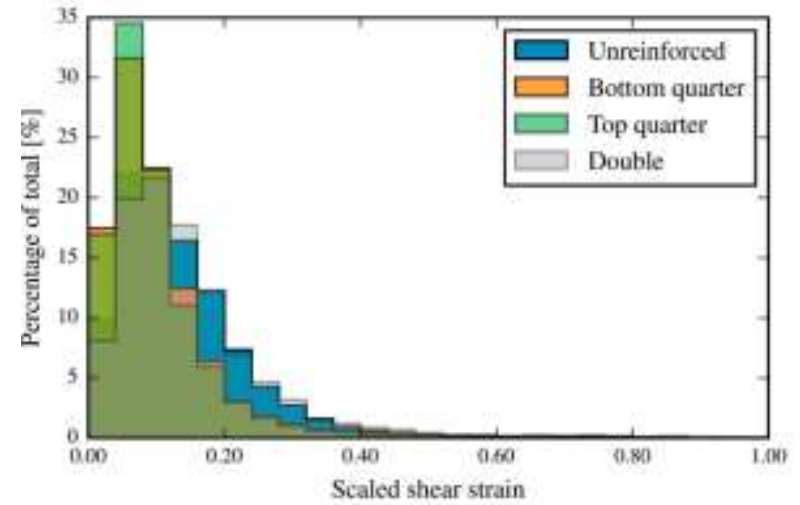

a)

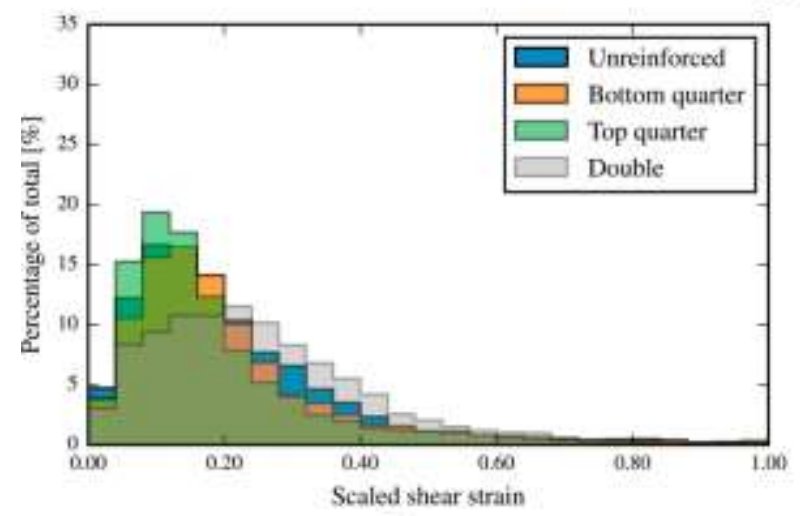

b)

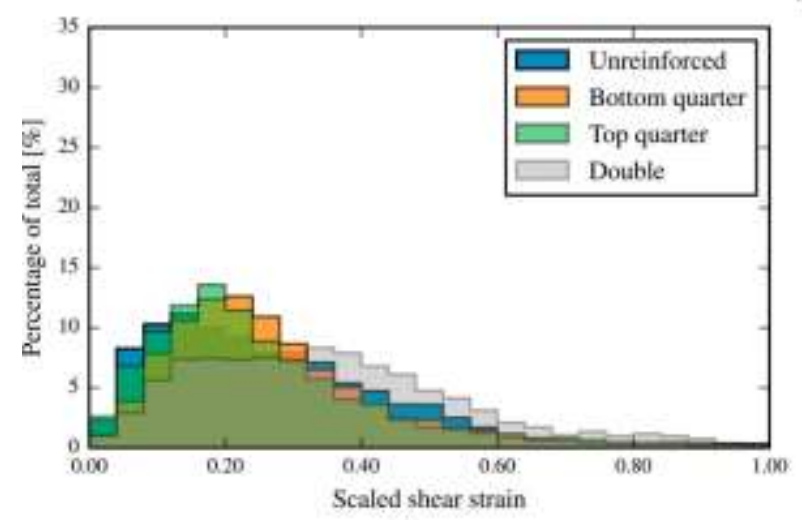

c)

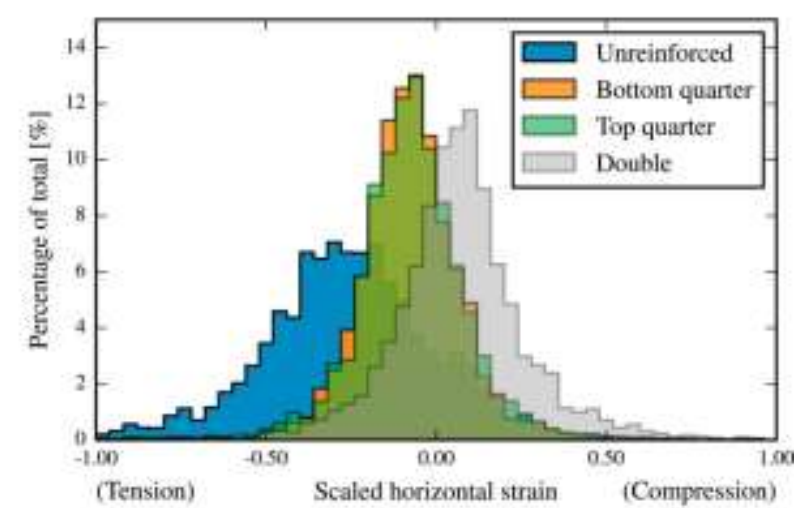

d)

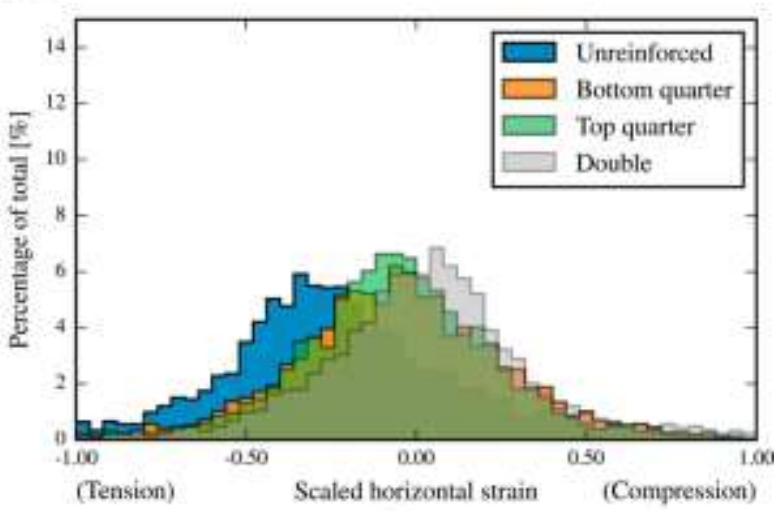

e)

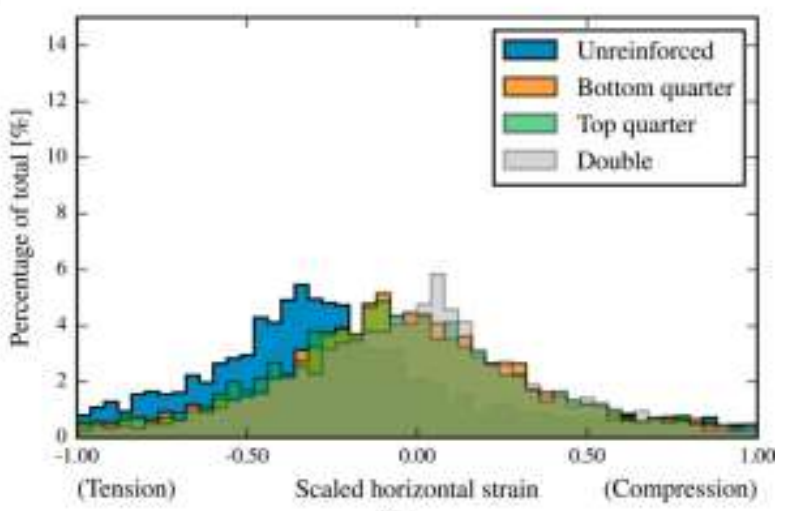

f)

Figure 10 - Histograms of the maximum shear $(a-c)$ and horizontal (d-e) strain distribution in the full liners. Stage I: a \& d, Stage II: b \& e and Stage III: c \& f.

\section{Histograms of strain distributions}

Additional insight into the behaviour of the model liners under settlement was gained by generating histograms of strain distribution from the calculated strain fields. In Figure 9 the process to generate these histograms is illustrated. Firstly, as illustrated in Figure 9a, the fraction of the liner that is at a given scaled shear strain, for example less than 0.4 , is measured. Secondly, the fraction is plotted on the histogram in Figure $9 \mathrm{~b}$ for the given strain magnitude. Consequently, it is possible to compare what fraction of the liner is at a given strain. In Figure 10 the distribution of both shear and 
horizontal strain across the full liners are presented as histograms. The horizontal strain was scaled in a similar manner as discussed for the shear strain in the previous section.

Consider the histograms of the shear strain fields over the full liners in Figure 10a-c. During Stages I, II \& III the shear strain distributions of the unreinforced, top-quarter reinforced and bottom-quarter reinforced liners were similar. However, the distribution of shear strain in the double reinforced liner is flatter than the others, indicating that a greater portion of the liner is strained, but at a lower intensity. Thus, the geogrid is effective in distributing the applied load across more of the clay liner, i.e. there is full compatibility between the geogrid and the clay even after significant differential settlement of the sand. Better compatibility implies that no separation between the geogrid and clay has occurred. Furthermore, a uniform shear strain distribution implies less strain concentrations, i.e. cracking, in the clay. Thus, with better geogrid-clay compatibility and less cracking after significant differential settlement of the sand, the permeability of the double reinforced liner will be lower than the other liners given the same reinforcement cost.

In Figure 10d the horizontal strain distributions for the bottom-quarter and top-quarter reinforced liners are symmetric around zero during Stage I. However, the horizontal strain distributions for the unreinforced liner is centred below zero. This indicates that, on average, the unreinforced liner was in tension during Stage 1. Similarly, micro-cracks were already identified in the unreinforced liner in Stage I (Table 2). Once micro-cracks were fully established in the bottom-quarter reinforced liner during Stage II (Figure 10e), and in the top-quarter reinforced liner during Stage III (Figure 10f), the mean values of these distributions also became tensile. However, the distribution of the doublereinforced liner remained centred in compression in Stages I, II and III indicating that, on average, the liner was in compression and that catastrophic crack growth had yet to occur. Thus, from the centrifuge tests it is shown that when crack growth is considered, equal distribution of the available reinforcement between the top-quarter and bottom-quarter of the liner is optimal.

\section{Practical considerations}

The recommendations of this study was laboratory based, informed by limited numerical analysis. Consequently, the practicality of installing geogrid reinforcement, especially double reinforcement, requires some consideration. From the centrifuge tests and numerical analyses done it was recommended that the reinforcement should be evenly distributed between the top and bottom quarters of the liner to limit crack growth. However, these two positions were selected as South African legislation requires that the $600 \mathrm{~mm}$ thick compacted clay liner in a composite barrier for a Class B (general waste) and Class A (hazardous waste) facility should be constructed of four $150 \mathrm{~mm}$ lifts. This allows for three different reinforcement positions within the liner: top-quarter, middle and bottom quarter. Should a liner be constructed using a different number of lifts, the reinforcement should be placed below the top-most and above the bottom-most lift. Thus, in the case of e.g. 10 lifts the geogrid should be placed in the top and bottom $10^{\text {th }}$ of the liner. This will ensure that the maximum tensile stress is distributed to the geogrid, while ensuring sufficient bond.

The selection of construction equipment also requires consideration. In some countries a fully penetrating padfoot roller is used when constructing a clay liner. The purpose of this roller is to penetrate through the top-most lift, into the one below, to compact the interface between the lifts. Consequently, any geogrid placed below only one layer of clay will be damaged by the roller. As an alternative construction strategy the surface of the clay layer below the geogrid can first be scarified with a standard padfoot roller. Thereafter, a large aperture geogrid (100 $\mathrm{cm}$ or larger) should be placed on top of the scarified surface. The clay should be specified such that all the voids around the geogrid will be filled when it is placed. Finally, the clay can be placed and compacted with a 
standard padfoot roller. As the pads of the padfoot roller are smaller than the apertures of the geogrid, adequate compaction through the geogrid will ensue. This proposed construction technique still has to be validated in full-scale tests.

Finally, some countries require that random samples of the compacted clay liner should be taken with a Shelby tube sampler as part of the quality assurance process. This sampling process has a high probability of damaging the geogrid. However, when considering that the main function of the geogrid is to stunt crack growth (mechanism 2), rather than providing physical reinforcement (mechanism 1), limited damage to the geogrid will not significantly compromise the performance of the system. In this case a geogrid with sufficient joint strength should be selected to ensure load is transferred around the damaged ribs.

\section{Conclusion and summary}

Numerical analyses and centrifuge modelling of reinforced liners were done to determine the optimal placement strategy for the reinforcement of clay liners below piggyback landfills. The results of the numerical analyses as reported by Marx \& Jacobsz (2016a and 2016b) illustrated that, in order to minimize the tensile strain in the liners, a double reinforcement strategy should be followed.

From the described centrifuge tests double reinforcement is also recommended as: 1) a greater magnitude of central settlement was required to induce micro cracks in the liner (see Figures 7 (, and 2 ) the cracks terminated at the level of the top geogrid (see Figure 8 ), and 3 ) the double reinforced liner remained on average in compression (see Figure 10). The centrifuge models did have the limitation that the model geogrids had a higher than typical stiffness. In practice, under modest differential settlement geogrid reinforcement will mostly serve to suppress crack growth, rather than increasing the stiffness of the system to reduce deflection.

Thus, from both the numerical analyses and the centrifuge tests it can be concluded that the reinforcement available should be placed immediately below the top-most layer and above the bottom-most layer in a clay liner for optimal performance should differential settlement of the underlying waste body occur. Some practical considerations for construction are presented which should be validated in practice.

\section{Acknowledgements}

The financial assistance of the Deutscher Akademischer Austausch Dienst (DAAD), the National Research Foundation of South Africa (NRF) and the Geosynthetics Interest Group of South Africa (GIGSA) are acknowledged by the first author. Opinions expressed and conclusions presented are those of the authors and are not necessarily to be attributed to the NRF or GIGSA.

\section{Appendix A}

The bending moment in a beam can be related to its deflection with the Bernoulli-Euler equation:

$$
M(x, y)=\frac{{ }^{2} y}{x^{2}} E I
$$

If a clay liner spans a void of width $l$, the deflected shape can be approximated by a beam of length $l$, fixed at both ends, subject to a distributed load $W$ equal to the self-weight of the beam:

$$
y(x, l)=\frac{W l}{24 E I}\left(\delta^{2}-2 \delta^{3}+\delta^{4}\right), \quad \delta=x / l
$$


Consequently:

$$
M(x, l)=\frac{W}{12} \cdot\left(l+6 x^{2} \cdot \frac{1}{l}-6 x\right)
$$

If a Gaussian shaped settlement trough is induced, similar to the surface depression caused by a tunnel (New \& O'Reilly, 1991), then the width of the void below the liner $(l)$ is:

$$
l \approx 5 i, \text { and } i=k^{*} \cdot z
$$

Where $k^{*}$ is an empirical factor related to the soil type and $z$ can be thought to be equivalent to the depth of the settlement trough, $\Delta$. Thus:

$$
l=5 k^{*} .
$$

And:

$$
M(x, l)=\frac{W}{12} \cdot\left(5 k^{*} \cdot \quad+6 x^{2} \cdot \frac{1}{5 k^{*} \cdot}-6 x\right)
$$

Consequently, at the centre of the liner $(x=0)$ :

$$
M \approx k \text {. }
$$

where $k$ is a constant.

\section{References}

Abu-Farsakh, M. Souci, G., Voyiadjis, G.Z., \& Chen, Q. 2012. Evaluation of Factors Affecting the Performance of Geogrid-Reinforced Granular Base Material Using Repeated Load Triaxial Tests. Journal of Geotechnical and Geoenvironmental Engineering, Vol 24, No. 1, pp $72-73$.

Alexview, D.A. 1997. Bridging a sink-hole by high-strength high-modulus geogrids. Proceedings of Geosynthetics '97, pp. 13-24.

Alfaro, M.C., Miura, N. \& Bergado, D.T. 1995. Soil-geogrid reinforcement interaction by pullout and direct shear tests. Geotechnical Testing Journal, Vol. 18, No. 2, pp 157-167.

Ajaz, A. \& Parry, R. 1975. Stress-strain behaviour two compacted clays in tension and compression. Géotechnique, Vol. 25, No. 3, pp. 495-512.

Archer, A. \& Heymann, G. 2015. Using small-strain stiffness to predict the settlement of shallow foundations on sand. Journal of the South African Institution of Civil Engineering, Vol 57, No 2, pp 2835.

Arora, J. 2004. Introduction to Optimum Design. Academic Press, p. 672.

Casagrande, A. 1932. Research on the Atterberg limits of soils. Public Roads, Vol. 13, No. 8, pp. 121136.

D6637-11, A. 2011. Standard Test Method for Determining Tensile Properties of Geogrids by the Single or Multi-Rib Tensile Method. Tech. rep. ASTM International.

Department of Environmental Affairs (DEA). 2013. National Environmental Management: Waste Act, 2008 (Act No 59 of 2008). Government Gazette: No. 36784:R.636 23 Aug. 
Edelmann, L., Katzenbach, R., Amann, P. \& Weiss, J. 1996. Large-scale deformation tests on soil layers for landfills. Proceedings of the 2nd International Congress on Environmental Geotechnics. Osaka, Japan, pp. 205-209

El-Fadel, M. \& Khoury, R. 2000. Modeling settlement in MSW landfills: a critical review. Critical Reviews in Environmental Science and Technology, Vol. 30, No. 3, pp. 327-361.

Giroud, J.-P. 1981. Designing with geotextiles. Matériaux et Constructions, Vol. 14, No. 82, pp. 257272.

Giroud, J.-P., Bonaparte, R., Beech, J. \& Gross, B. 1990. Design of soil layer-geosynthetic systems overlying voids. Geotextiles and Geomembranes, Vol. 9, No. 1, pp. 11-50.

Gourc, J., Camp, S., Viswanadham, B. \& Rajesh, S. 2010. Deformation behavior of clay cap barriers of hazardous waste containment systems: Full-scale and centrifuge tests. Geotextiles and Geomembranes, Vol. 28, No. 3, pp. 281-291.

Griffith, A. A. 1920. The Phenomena of Rupture and Flow in Solids. Philosophical Transactions of the Royal Society A: Mathematical, Physical and Engineering Sciences, Vol. 221, No. 582-593, pp. 163198.

Hubert, M. \& Vandervieren, E. 2008. An adjusted boxplot for skewed distributions. Computational Statistics and Data Analysis, Vol. 52, No. 12, pp. 5186-5201.

Jacobsz, S., Kearsley, E. \& Kock, J. 2014. The geotechnical centrifuge facility at the University of Pretoria. Proceedings of the 8th International Conference on Physical Modelling in Geotechnics 2014. Perth, Australia: CRC Press.

Jessberger, H. \& Stone, K. 1991. Subsidence effects on clay barriers. Géotechnique, Vol. 41, No. 2, pp. 185-194.

Karihaloo, B. 1995. Fracture mechanics \& structural concrete. Longman Scientific \& Technical.

Koutsourais, M., Sprague, C. \& Pucetas, R. 1991. Interfacial friction study of cap and liner components for landfill design. Geotextiles and Geomembranes, Vol. 10, No. 5-6, pp. 531-548.

Kuo, C. \& Hsu, T. 2003. Traffic induced reflective cracking on pavements with geogrid-reinforced asphalt concrete overlay. Proceedings of the 82th Annual Meeting at the Transportation Research Board.

Lehane, B., O'Loughlin, C., Gaudin, C. \& Randolph, M. 2009. Rate effects on penetrometer resistance in kaolin. Géotechnique, Vol. 59, No. 1, pp. 41-52.

Marx, D. \& Jacobsz, S. 2016a. Geometric factors influencing the optimal position of geogrid reinforcement. Proceedings of the 6th European Geosynthetics Congress. Ljubljana, Slovenia, pp. $1455-1466$.

Marx, D. \& Jacobsz, S. 2016b. Optimal geogrid reinforcement of clay liners. Proceedings of the First Southern African Geotechnical Conference. Ed. by S. Jacobsz. CRC Press.

Mishra, D., Qian, Y., Kazmee, H. \& Tutumluer, E. 2014. Investigation of Geogrid-Reinforced Railroad Ballast Behavior Using Large-Scale Triaxial Testing and Discrete Element Modeling. Transportation Research Record: Journal of the Transportation Research Board, No. 2462, pp 98-108. 
Mousavi, S.H., Gabr, M. \& Borden, R., 2017. Optimum location of the geogrid reinforcement in unpaved road. Canadian Geotechnical Journal, Vol. 54. , pp 1047 - 1054.

New, B. \& O'Reilly, M. 1991. Tunnelling induced ground movements; predicting their magnitude and effects. Proceedings of the 4th International Conference on Ground Movements and Structures, invited review paper, Cardiff, Pentech Press, London, pp. 671-697.

Palmeira, E. \& Milligan, G. 1989. Scale and other factors affecting the results of pull-out tests of grids buried in sand. Geotechnique, Vol. 39, No. 3, pp. 511-524.

Palmeira, E. \& Viana, H. 2003. Effectiveness of geogrids as inclusions in cover soils of slopes of waste disposal areas. Geotextiles and Geomembranes, Vol. 21, No. 5, pp. 317-337.

Plé, O., Manicacci, A., Gourc, J. \& Camp, S. 2012. Flexural behaviour of a clay layer: experimental and numerical study. Canadian Geotechnical Journal, Vol. 49, No. 4, pp. 485-493.

Rajesh, S. \& Viswanadham, B. 2009. Evaluation of geogrid as a reinforcement layer in clay based engineered barriers. Applied Clay Science, Vol. 46, No. 2, pp. 153-165.

Rajesh, S. \& Viswanadham, B. 2011. Hydro-mechanical behavior of geogrid reinforced soil barriers of landfill cover systems. Geotextiles and Geomembranes, Vol. 29, No. 1, pp. 51-64.

Rajesh, S. \& Viswanadham, B. 2012b. Centrifuge Modeling and Instrumentation of Geogrid-

Reinforced Soil Barriers of Landfill Covers. Journal of Geotechnical and Geoenvironmental

Engineering, Vol. 138, No. 1, pp. 26-37.

Rousseeuw, P. \& Hubert, M. 2011. Robust statistics for outlier detection. Wiley Interdisciplinary Reviews: Data Mining and Knowledge Discovery, Vol. 1, No. 1, pp. 73-79.

Schofield, A. \& Wroth, P. 1968. Critical State Soil Mechanics. McGraw-Hill, London.

Shinoda, M. \& Bathurst, R. 2004. Lateral and axial deformation of PP, HDPE and PET geogrids under tensile load. Geotextiles and Geomembranes, Vol. 22, No. 4, pp. 205-222.

Stathas, D., Wang, J.P. \& Ling, H.I. 2017. Model geogrids and 3D printing. Geotextiles and Geomembranes, Vol 45, pp. $688-696$.

Stanier, S., Blaber, J, Take,W. A. \& White, D. 2015. Improved image-based deformation measurement for geotechnical applications. Canadian Geotechnical Journal, Vol. 13, No. October 2015, pp. 1-35.

Thusyanthan, N.I., Take, W.A., Madabhushi, S.P.G. \& Bolton, M.D. 2007. Crack initiation in clay observed in beam bending. Géotechnique, Vol 57, No 7, pp. $581-594$

Turner, C. \& Kolednik, O. 1994. A micro and macro approach to the energy dissipation rate model of stable ductile crack growth. Fatigue \& Fracture of Engineering Materials \& Structures, Vol. 17, No. 9, pp. 1089-1107

Viswanadham, B. 1996. Geosynthetic reinforced mineral sealing layers of landfills. Phd Thesis. Bochum, Germany: Ruhr University.

Viswanadham, B. \& Mahesh, K. 2002. Modelling deformation behaviour of clay liners in a small centrifuge. Canadian Geotechnical Journal, Vol. 39, No. 6, pp. 1406-1418. 
Viswanadham, B. \& König, D. 2004. Studies on scaling and instrumentation of a geogrid. Geotextiles and Geomembranes, Vol. 22, No. 5, pp. 307-328.

Viswanadham, B. \& Jessberger, H. 2005. Centrifuge Modeling of Geosynthetic Reinforced Clay Liners of Landfills. Journal of Geotechnical and Geoenvironmental Engineering, Vol. 131, No. 5, pp. 564574.

Viswanadham, B. \& Muthukumaran, A. 2007. Influence of geogrid layer on the integrity of compacted clay liners of landfills. Soils and Foundations, Vol. 47, No. 3, pp. 517-532.

White, D., Take, W. \& Bolton, M.D. 2003. Soil deformation measurement using particle image velocimetry (PIV) and photogrammetry. Geotechnique, Vol. 53, No. 7, pp. 619-631.

Zekkos, D., Fei, X., Grizi, A., \& Athanasopoulos, G.A. 2017. Response of Municipal Solid Waste to Mechanical Compression. Journal of Geotechnical and Geoenvironmental Engineering, Vol 143, No 3.

Zornberg, J. G., J. K. Mitchell, \& N. Sitar. 1997. Testing of reinforced slopes in a geotechnical centrifuge. Geotechnical Testing Journal, Vol 20 No 4, pp. 470-480.

Zornberg, J. G., Roodi, G.H. \& Gupta, R. 2017. Stiffness of Soil-Geosynthetic Composite under Small Displacements: I. Model Development. Journal of Geotechnical and Geoenvironmental Engineering, Vol. 143, No. 10. 